\title{
Nutrient-starved, non-replicating Mycobacterium tuberculosis requires respiration, ATP synthase and isocitrate lyase for maintenance of ATP homeostasis and viability
}

\author{
Martin Gengenbacher, Srinivasa P. S. Rao, Kevin Pethe and Thomas Dick \\ Novartis Institute for Tropical Diseases Pte Ltd, 10 Biopolis Road, \#05-01 Chromos, 138670, \\ Singapore
}

Correspondence

Martin Gengenbacher

martin.gengenbacher@

novartis.com

Received 27 July 2009

Revised 23 September 2009

Accepted 1 October 2009

\begin{abstract}
The ability of Mycobacterium tuberculosis to persist in its human host despite extensive chemotherapy is thought to be based on subpopulations of non-replicating phenotypically drugresistant bacilli. To study the non-growing pathogen, culture models that generate quiescent organisms by either oxygen depletion in nutrient-rich medium (Wayne model) or nutrient deprivation in oxygen-rich medium (Loebel model) have been developed. In contrast to the energy metabolism of Wayne bacilli, little is known about Loebel bacilli. Here we analysed M. tuberculosis under nutrient-starvation conditions. Upon shifting to the non-replicating state the pathogen maintained a fivefold reduced but constant intracellular ATP level. Chemical probing of the $F_{0} F_{1}$ ATP synthase demonstrated the importance of this enzyme for ATP homeostasis and viability of the nutrient-starved organism. Surprisingly, the specific ATP synthase inhibitor TMC207 did not affect viability and only moderately reduced the intracellular ATP level of nutrient-starved organisms. Depletion of oxygen killed Loebel bacilli, whereas death was prevented by nitrate, suggesting that respiration and an exogenous electron acceptor are required for maintaining viability. Nutrient-starved bacilli lacking the glyoxylate shunt enzyme isocitrate lyase failed to reduce their intracellular ATP level and died, thus establishing a link between ATP control and intermediary metabolism. We conclude that reduction of the ATP level might be an important step in the adaptation of $M$. tuberculosis to non-growing survival.
\end{abstract}

\section{INTRODUCTION}

Mycobacterium tuberculosis claims approximately 2 million lives annually (Harries \& Dye, 2006). A major objective of ongoing drug discovery efforts is to shorten chemotherapy from the current 6 months to 2 months or less (Williams \& Duncan, 2007). Popular working models suggest that unfavourable micro-environmental conditions inside the human lesions, such as oxygen and nutrient limitation, are external triggers that terminate growth of subpopulations of tubercle bacilli and render them phenotypically resistant to drugs, thus explaining the long treatment periods needed to cure the disease (Dick, 2001; Wayne \& Sohaskey, 2001). Indeed, in vitro models using oxygen depletion in nutrient-rich medium (Wayne model) or nutrient starvation in oxygen-rich medium (Loebel model) have demonstrated the survival of M. tuberculosis for extended periods in a non-replicating and drug-tolerant state (Betts

Abbreviations: DCCD, $N, N^{\prime}$-dicyclohexylcarbodiimide; Icl, isocitrate lysase; Ndh, NADH dehydrogenase; r.l.u., relative light unit.

A supplementary figure is available with the online version of this paper. et al., 2002; Wayne \& Hayes, 1996). Recently, the energy metabolism of hypoxic non-replicating tubercle bacilli has been characterized. The intracellular ATP level of Wayne bacilli is reduced fivefold compared to that of exponentially growing bacteria (Koul et al., 2008; Rao et al., 2008). The $\mathrm{F}_{0} \mathrm{~F}_{1}$ ATP synthase and the respiratory enzyme NADH dehydrogenase (Ndh)-2, but not Ndh-1, were shown to be essential for the survival of oxygen-starved bacilli (Koul et al., 2008; Rao et al., 2008). Nutrient-starved bacilli have been less well characterized. Loebel et al. (1933a) showed that survival of the tubercle bacillus under those conditions was accompanied by a drastic reduction of respiration, indicating a very low metabolic rate or even metabolic inactivity. This was further supported by more recent transcriptomic analyses, which demonstrated a strong downregulation of metabolic genes, including those encoding enzymes of the respiratory machinery (Betts et al., 2002). Animal models as well as human lesion studies provided evidence for the presence of Loebel-like bacilli in vivo: the stringent response essential for adaptation to nutrient starvation in vitro (Betts et al., 2002; Primm et al., 2000) was shown to be required for persistence of bacilli in 
the tuberculosis mouse model (Dahl et al., 2003). Nutrientstarvation-induced transcripts of the genes Rv2557 and Rv2558 were detected in human granulomas (Betts et al., 2002, Fenhalls et al., 2002). However, genetic analyses showed that neither of these genes was required for survival of bacilli in the Loebel culture model (Gordhan et al., 2006). In the current study, we have analysed the metabolism of nutrient-starved M. tuberculosis in vitro. We focused on energy metabolism, respiration and the intracellular ATP level, since these were found to be critical determinants for viability of the hypoxic non-replicating pathogen (Rao et al., 2008).

\section{METHODS}

Chemicals. Rifampicin, streptomycin, isoniazid, $N, N^{\prime}$-dicyclohexylcarbodiimide (DCCD), tyloxapol and Tween 80 were purchased from Sigma-Aldrich. Moxifloxacin was obtained from Chempacific and the compound TMC207 was synthesized as described elsewhere (Andries et al., 2005). Middlebrook 7H9, Middlebrook OADC Enrichment, Middlebrook 7H11 Agar, Dubos and Dubos medium albumin were purchased from Becton Dickinson.

Growth, oxygen depletion and nutrient starvation. $M$. tuberculosis H37Rv (American Type Culture Collection \#27294) was grown in 11 roller bottles (Corning) containing Middlebrook 7H9 medium supplemented with $0.5 \%$ albumin, $0.2 \%$ glucose, $0.085 \%$ $\mathrm{NaCl}, 0.2 \%$ glycerol and $0.05 \%$ Tween 80 , at 2 r.p.m. and $37{ }^{\circ} \mathrm{C}$. For nutrient-starvation experiments, exponentially growing bacilli at an $\mathrm{OD}_{600}$ of $0.1-0.2$ were harvested by centrifugation $(2500 \mathrm{~g}, 10 \mathrm{~min})$, washed twice with PBS (Invitrogen) supplemented with $0.025 \%$ Tween 80 (PBST) and diluted to a final $\mathrm{OD}_{600}$ of $0.1 ; 50 \mathrm{ml}$ of this suspension was transferred into a 11 roller bottle and starved for 14 days or longer $\left(37^{\circ} \mathrm{C}, 2\right.$ r.p.m.). To prove that Tween 80 was not causing the observed death of the icl loss-of-function mutant, we replaced it by the non-hydrolysable detergent tyloxapol. However, this did not affect the result. Oxygen starvation was carried out as described previously (Lim et al., 1999; Wayne \& Hayes, 1996). Briefly, H37Rv was maintained in Dubos liquid medium containing $0.03 \%$ Tween 80 and $10 \%$ Dubos medium albumin. Hypoxic nonreplicating bacilli were generated under conditions of slow stirring (130 r.p.m.) in sealed glass tubes. After cultures became hypoxic, which was visualized by decolorization of methylene blue in a control tube, usually occurring on day 12 , they were further starved of oxygen for 8 days until experimental use.

Determination of bactericidal concentrations. DCCD was dissolved in ethanol, whereas all other compounds were dissolved in $90 \%$ DMSO to prepare stock solutions. The cidal activity of compounds against growing and non-growing bacilli was determined by exposing an initial inoculm of $\sim 10^{6}$ c.f.u. $\mathrm{ml}^{-1}$ for 5 days to the drugs in a 96-well clear flat-bottom plate (Corning) at $37^{\circ} \mathrm{C}$ (standing). After that, c.f.u. were determined by plating appropriate dilutions of the cultures on Middlebrook $7 \mathrm{H} 11$ agar plates supplemented with $10 \%$ Middlebrook OADC Enrichment and $0.2 \%$ glycerol. The concentration of a compound that reduced c.f.u. by $90 \%$ was defined as the minimal bactericidal concentration ${ }_{90}$ $\left(\mathrm{MBC}_{90}\right)$ for exponentially growing bacilli, and as the Wayne cidal concentration $_{90}\left(\mathrm{WCC}_{90}\right)$ and Loebel cidal concentration ${ }_{90}\left(\mathrm{LCC}_{90}\right)$ for the two states of non-growing bacilli. Oxygen- and nutrientstarved stationary-phase cultures used for drug sensitivity determination were 20 and 14 days old respectively.

Determination of the intracellular ATP level. Bacilli collected from various experiments were immediately heat-inactivated $\left(90{ }^{\circ} \mathrm{C}\right.$,
$20 \mathrm{~min}$ ) and stored at $-80{ }^{\circ} \mathrm{C}$ until further use. The intracellular ATP was quantified using the BacTiter-Glo Microbial Cell Viability Assay kit from Promega according to the manufacturer's instructions. Briefly, $25 \mu \mathrm{l}$ of each heat-inactivated sample was mixed with an equal volume of freshly prepared BacTiter-Glo reagent in a white flatbottom 96-well plate (Corning) and incubated for $5 \mathrm{~min}$ in the dark. The emitted luminescence was detected using a Safire ${ }^{2}$ plate reader (Tecan instruments) and displayed as relative light units (r.l.u.); the readings were directly correlated with the ATP content of the respective sample. All experiments were standardized using ATP solutions ranging from 0.1 to $100 \mathrm{nM}$ as internal control. The absolute amount of ATP per bacterium was calculated by dividing mol ATP $\mathrm{ml}^{-1}$ by c.f.u. $\mathrm{ml}^{-1}$.

Anaerobic shift of nutrient-starved $\boldsymbol{M}$. tuberculosis. Bacilli were nutrient-starved for 14 days as described above. Aliquots of $1 \mathrm{ml}$ were transferred into a 24-well plate and placed in a sealed container. Oxygen was removed from the gas phase by an AnaeroGen absorbing gas pack (Oxoid) and monitored by a methylene blue strip. Control wells containing either PBST or bacilli plus PBST were supplemented with methylene blue (final concentration $1.5 \mu \mathrm{g} \mathrm{ml}^{-1}$ ) to observe the oxygen consumption of the pathogen. In order to study a more rapid shift to hypoxia, the experiment was repeated similarly, but bacilli were centrifuged $(2500 \mathrm{~g}, 10 \mathrm{~min})$ and resuspended in oxygen-free PBST prior to applying anaerobic conditions. The samples taken during the course of experiments were plated on 7H11 agar for c.f.u. estimation.

Gene knockout of icl and complementation. The icl (Rv0467) gene represents the only known functional copy encoding an isocitrate lyase in the $\mathrm{H} 37 \mathrm{Rv}$ genome (Honer $\mathrm{Zu}$ Bentrup et al., 1999). The icl gene was interrupted by the allelic-exchange method using the plasmid pYUB854 (Bardarov et al., 2002). Briefly, the flanking regions of the gene were amplified by PCR using specific oligonucleotides (5'-region: 5'-TACTCGAGCTTGTCGGCACATCCAGGACA-3' and 5'-GCAAGCTTTCCTCGGCGGAGTAGGTGCGG-3'; 3'-region: 5'-GTTCTAGAGGCTACTTCGACCGGATTGCC$3^{\prime}$ and $5^{\prime}$-CACTTAAGGGGGGCCGTTCATACACTTCA-3') and inserted upstream and downstream of the hygromycin $B$ selection cassette of pYUB854. Additionally, the sacB-lacZ cassette was excised from pGOAL17 (Parish \& Stoker, 2000) and ligated into the PacI sites of pYUB854, containing the $5^{\prime}$ and $3^{\prime}$ flanks of the $i c l$ locus.

The loss-of-function mutant strain $\Delta i c l$ was complemented with the functional icl gene using the integrative vector pMV306 (Stover et al., 1991). The gene, including a $274 \mathrm{bp}$ upstream region, which might contain the native promoter, was amplified by PCR using the specific oligonucleotides 5'-ATAAAGCTTCCGGATCCGCAGGACGTCGA-

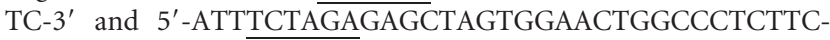
GGTGG-3' and inserted into pMV306, containing an attachment site and an integrase gene derived from mycobacteriophage L5, which allows the integration of the vector into the genome of M. tuberculosis by site-specific recombination (Lee et al., 1991). In contrast to the pMV306 complementation vector, the final plasmid preparation of the pYUB854 knockout construct was UV-irradiated prior to electroporation into M. tuberculosis (Hinds et al., 1999).

\section{RESULTS}

\section{Nutrient-starved bacilli maintain viability and are extremely drug tolerant}

To generate nutrient-starved non-replicating bacilli, cultures of exponentially growing $M$. tuberculosis $\mathrm{H} 37 \mathrm{Rv}$ were washed, resuspended in PBS and incubated in rolling roller 
bottles (Betts et al., 2002, Loebel et al., 1933a, b; Xie et al., 2005). Fig. 1 shows that the tubercle bacillus maintained full viability in this aerated, nutrient-starved stationary phase. The upper part of Table 1 summarizes the extraordinary tolerance of nutrient-starved, non-growing bacilli to antimycobacterials (Xie et al., 2005). Rifampicin, streptomycin, moxifloxacin and isoniazid, all highly bactericidal for growing bacilli, had little or no effect on the viability of Loebel bacilli. Table 1 also shows that these drugs (with the exception of isoniazid) had a reduced but still significant cidal activity on hypoxic non-replicating $M$. tuberculosis. The dramatically higher level of drug tolerance of Loebel bacilli compared to Wayne bacilli suggests a more 'spore-like', metabolically inactive state for the nutrientstarved form of the tubercle bacillus.

\section{The intracellular ATP level of nutrient-starved $\boldsymbol{M}$. tuberculosis is reduced fivefold}

Recent studies of the tubercle bacillus in the Wayne culture model demonstrated that the organism reduces its intracellular ATP level fivefold when it shifts down to a hypoxic, non-replicating state (Koul et al., 2008; Rao et al., 2008). To determine whether the ATP level reduction also occurs upon the nutrient-starvation-induced shiftdown to the non-growing state, we monitored the intracellular ATP of bacilli in Loebel cultures over time. Fig. 1 shows that the nutrient-starvation-induced shiftdown to the stationary

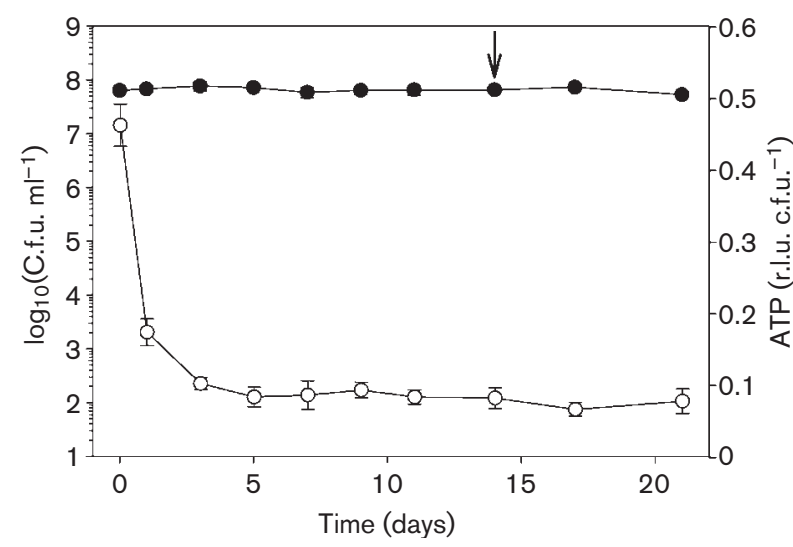

Fig. 1. Viability and intracellular ATP level of $M$. tuberculosis during nutrient starvation. C.f.u. $\mathrm{ml}^{-1}(\mathbf{O})$ and intracellular ATP level [relative light units (r.l.u.) c.f.u. ${ }^{-1}(\bigcirc)$ ] were monitored over 21 days. Exponentially growing bacilli were washed twice, and nutrient-starved in PBS in roller bottles at 2 r.p.m. $/ 37^{\circ} \mathrm{C}$. The experiment was repeated four times and samples were taken in triplicate. Mean values and standard deviations of one representative experiment are shown. Two of the experiments included a 42 day time point. Viability and ATP level remained the same after 14 and 42 days (data not shown). The arrow (14 days) indicates the age of the 'standard' Loebel culture, used for drug susceptibility determinations (Table 1), probing with DCCD and TMC207 (Fig. 2) or respiration experiments (Fig. 3).
Table 1. Drug susceptibility of growing versus oxygen- and nutrient-starved non-growing M. tuberculosis

Minimum bactericidal concentration $\left(\mathrm{MBC}_{90}\right)$, Wayne cidal concentration $\left(\mathrm{WCC}_{90}\right)$ and Loebel cidal concentration $\left(\mathrm{LCC}_{90}\right)$ were defined as the concentration $(\mu \mathrm{M})$ of the compound that reduced c.f.u. of the initial inoculum by $90 \%$ after 5 days of exposure under the respective culture conditions.

\begin{tabular}{|lccc|}
\hline \multirow{2}{*}{ Drug } & $\begin{array}{c}\text { Exponentially } \\
\text { growing } \\
\text { MBC }_{\mathbf{9 0}}\end{array}$ & \multicolumn{2}{c|}{ Non-growing } \\
\cline { 3 - 4 } & 0.01 & WCC $_{\mathbf{9 0}}$ & LCC $_{\mathbf{9 0}}$ * \\
\hline Rifampicin & 0.125 & 0.5 & 25 \\
Streptomycin & 0.125 & 6 & $>100$ \\
Moxifloxacin & 0.4 & $>100$ & $>100$ \\
Isoniazid & 5 & 2.5 & $>100$ \\
\hline TMC207 & $>500$ & 80 & 20 \\
DCCD & & & \\
\hline
\end{tabular}

${ }^{*} \mathrm{LCC}_{90}$ was determined using cultures starved for 14 days from nutrients (arrow in Fig. 1). Identical LCC $_{90}$ results were obtained from bacilli starved for 42 days (data not shown).

phase was accompanied by a fivefold drop in the intracellular ATP level (from $3.39 \pm 0.21 \times 10^{-18}$ to $0.59 \pm 0.10 \times 10^{-18} \mathrm{~mol} \mathrm{c.f.u.}^{-1}$ ), identical to the reduction observed for hypoxic non-replicating bacilli (Rao et al., 2008). This result suggests that ATP level reduction could be a general metabolic theme for adaptation of $M$. tuberculosis to quiescent survival.

\section{Loebel bacilli require the $F_{0} F_{1}$ ATP synthase to maintain ATP homeostasis and viability}

Previous reports demonstrated the bactericidal activity of the ATP synthase inhibitors TMC207 and DCCD against exponentially growing as well as hypoxic non-replicating bacilli (Andries et al., 2005; Koul et al., 2007; Rao et al., 2008). These findings established that the ATP synthase is essential for the survival of both physiological states of $M$. tuberculosis (Koul et al., 2008; Rao et al., 2008). DCCD inhibits the ATP synthase by a covalent modification of the $\mathrm{F}_{0}$ subunit (Cattell et al., 1971; Ovchinnikov et al., 1982). To determine whether the ATP synthase activity is required for survival of nutrient-starved bacilli, this non-growing form of the pathogen was exposed to both inhibitors. Fig. 2(a) shows that TMC207 did not affect viability of Loebel bacilli. However, we observed a concentration-dependent reduction of the intracellular ATP level in those organisms (Fig. 2b). In contrast to this result, DCCD reduced the number of viable organisms about 10 -fold at a concentration of $20 \mu \mathrm{M}$ (Fig. 2a) and reduced the intracellular ATP level about 3.5-fold (Fig. 2b). Interestingly, at a concentration of $10 \mu \mathrm{M}$ DCCD, viability was not affected, but the ATP level was reduced, comparable to the reduction observed for $100 \mu \mathrm{M}$ TMC207. We could not test higher 
(a)

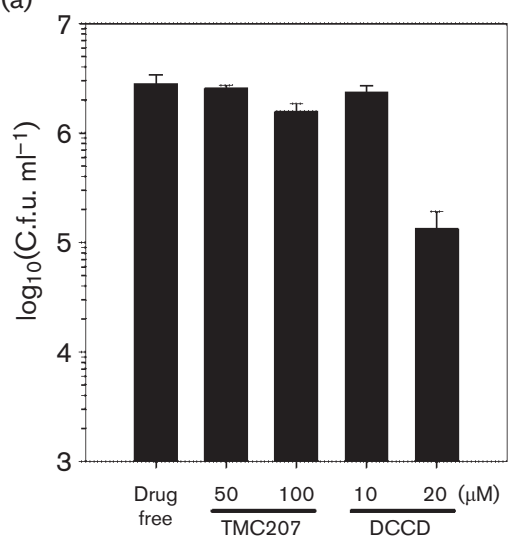

(b)

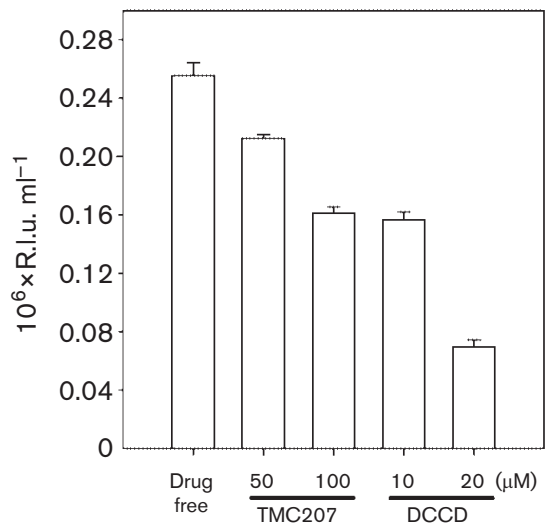

Fig. 2. $F_{0} F_{1}$ ATP synthase activity is required for ATP homeostasis and survival of nutrientstarved M. tuberculosis. Loebel bacilli were exposed to varying concentrations of the $F_{0} F_{1}$ ATP synthase inhibitors TMC207 and DCCD. After 5 days, viability (a) and intracellular ATP levels (b) were determined. The experiment was carried out three times in triplicate and results are given as means and standard deviations. concentrations of TMC207 because of its low solubility; doubling of the drug exposure time did not result in further depletion of intracellular ATP (data not shown). A comparison of the bactericidal activity of TMC207 and DCCD in replicating and non-replicating bacilli is shown at the bottom of Table 1. Together, these results demonstrated that nutrient-starved $M$. tuberculosis requires ATP synthase to maintain ATP homeostasis and full viability. However, Loebel bacilli turned out to be less sensitive to ATP depletion compared to Wayne bacilli (Rao et al., 2008). This might point to a extraordinarily low metabolic activity of Loebel bacilli.

\section{Respiration is required for survival in the absence of external nutrients}

Previously, a drastic reduction of respiration in $M$. tuberculosis within the first $96 \mathrm{~h}$ of nutrient starvation has been reported (Betts et al., 2002; Loebel et al., 1933a). To determine whether Loebel bacilli indeed do not consume any oxygen, we transferred a nutrient-starved culture into an anaerobic atmosphere and monitored the consumption of oxygen remaining in the culture by methylene blue decolorization. Fig. 3(a) shows that wells containing nutrient-starved bacilli were decolorized after 6 days, indicating continuous respiratory activity. Negative control wells without bacteria did not decolorize during the analysed period of time (Fig. 3a). To determine whether the observed continued oxygen consumption is required for survival of Loebel bacilli, nutrient-starved cultures were harvested and resuspended in oxygen-free PBST. Fig. 3(b) shows that exposure of nutrient-starved bacilli to anaerobic conditions killed the bacteria, suggesting that $M$. tuberculosis requires respiration and an exogenous electron acceptor for survival. To confirm this, we added the alternative electron acceptor nitrate to hypoxic nutrient-starved cultures and monitored their viability. Nitrate indeed rescued Loebel bacilli under anaerobic conditions (Fig. 3b), confirming their need for continuous respiration and demonstrating their ability to carry out nitrate respiration. Taken together, these results demonstrate that nutrient-starved, non-growing bacilli require continuous respiration for survival, and that they tolerate both oxygen and nitrate as exogenous electron acceptors.

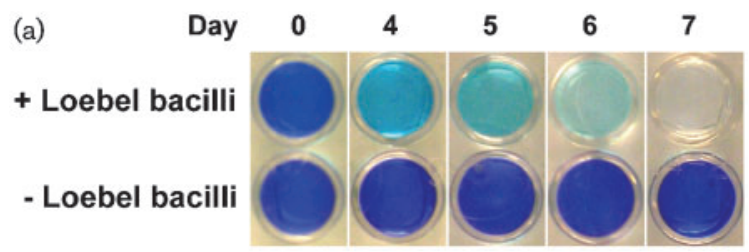

(b)

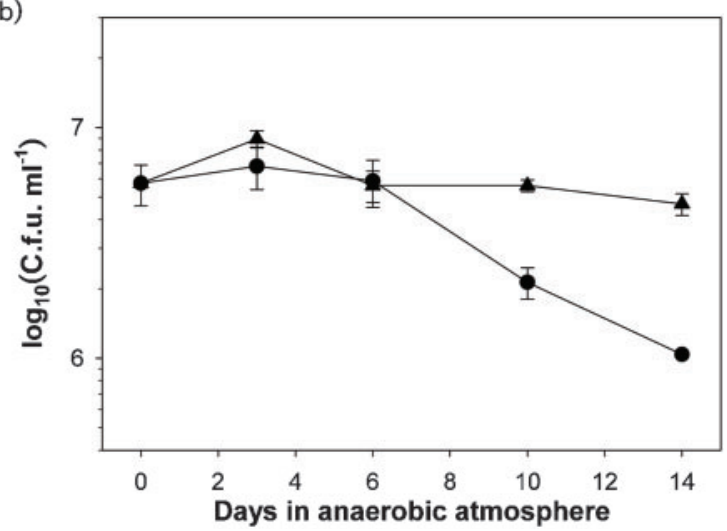

Fig. 3. Loebel bacilli continuously respire and depend on an exogenous electron acceptor. (a) Upper row: Cultures were nutrient-starved for 14 days (arrow in Fig. 1) and then shifted into an anaerobic atmosphere (day 0). The presence of oxygen left in the liquid phase was monitored by decolorization of methylene blue (standing culture, $37^{\circ} \mathrm{C}$ ). Methylene blue decolorized after 6 days. Lower row: Control wells with methylene blue - but without Loebel bacilli - in an anaerobic atmosphere. (b) 14 day-old nutrientstarved cultures were resuspended in oxygen-free starvation medium prior to the shift into a hypoxic atmosphere. Loebel bacilli lost viability upon exposure to hypoxia ( $)$, whereas supplementation with $10 \mathrm{mM}$ nitrate prevented death $(\boldsymbol{\Delta})$. 


\section{The icl gene is required for non-growing survival in the Loebel model}

Nutrient deprivation results in a major shutdown of global gene expression. Remarkably, the transcript level of the glyoxylate shunt enzyme isocitrate lysase (Icl) was not reduced in Loebel bacilli (Betts et al., 2002). Furthermore, it had been demonstrated that Icl function is essential for survival of the pathogen in the chronic tuberculosis mouse model (McKinney et al., 2000). Together, these observations prompted us to hypothesize that Icl might play a role in the adaptation of the pathogen when nutrients are deprived. The icl loss-of-function mutant of $M$. tuberculosis indeed dies in the Loebel model (Fig. 4a). Viability was fully restored when the mutant strain was complemented with a wild-type copy of $i c l$. Fig. 4(b) shows the effect of the icl loss-of-function mutation on the kinetics of the intracellular ATP level reduction upon shiftdown to the non-growing state. Whereas wild-type $M$. tuberculosis reduced its ATP level rapidly upon the shift from growth in rich medium to carbon starvation, the $i c l$ mutant failed
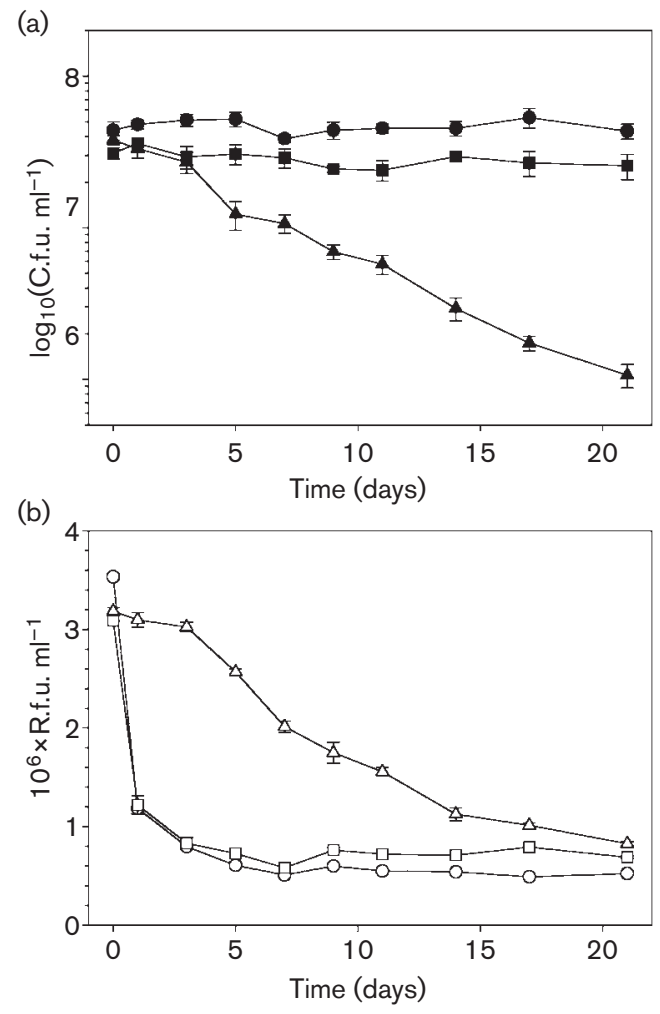

Fig. 4. The glyoxylate shunt enzyme isocitrate lyase (Icl) is required for survival of nutrient-starved non-growing tubercle bacilli. Wild-type M. tuberculosis (circles), the icl loss-of-function strain $\Delta i c l$ (triangles) and the $\Delta i c l$ strain complemented with the wild-type icl gene (squares) were nutrient-starved at $37^{\circ} \mathrm{C}$. Viability (a) and the intracellular ATP level (b) were monitored over 21 days. Mean values and standard deviations of triplicate samples from one experiment out of three are displayed. to reduce its intracellular ATP concentration. These results suggest a link between Icl as part of the intermediary metabolism, intracellular ATP level regulation and nonreplicating survival.

\section{DISCUSSION}

Non-growing bacteria are tolerant to antimicrobials that, in general, target functions essential for growth. $M$. tuberculosis is no exception. Therefore, the working model that prolonged chemotherapy is required to cure tuberculosis could be due to populations of non-replicating bacilli is attractive. The two main in vitro culture models employed to generate and study non-growing bacilli are based on oxygen and nutrient starvation, respectively (Betts et al., 2002; Wayne \& Hayes, 1996). Which of these two stationary-phase culture systems, if any, mimics bacilli present in human lesions remains to be determined. There is evidence that $M$. tuberculosis in vivo suffers nutrient limitation. The staining properties and morphology of bacilli starved in distilled water or isolated from lesions were shown to be comparable, but significantly different from the respective features of growing cultures (Nyka, 1974). More recent studies demonstrated that some lesions in animal models provide a hypoxic microenvironment for the residing tubercle bacillus (Via et al., 2008). However, it appears likely that neither of the models represents the precise conditions and metabolic states of non-replicating bacilli in vivo since the specific nutrient and oxygen parameters in both systems were chosen arbitrarily. The two models employ directly opposite culture conditions to trigger the shiftdown of $M$. tuberculosis to a non-growing state: 'no oxygen/rich medium' in the Wayne model versus 'oxygen saturation/no nutrients' in the Loebel model. Hence, it is conceivable that metabolic functions and adaptations identified to be essential for the survival of both these artificial non-growing states of M. tuberculosis might represent processes that are of general importance for the survival of non-replicating bacilli. At least to some extent those processes might be independent of the specific environmental conditions chosen in the model systems. With this in mind, we analysed the intracellular ATP level and the respiratory/oxidative phosphorylation functions that were previously shown to be essential for oxygenstarved bacilli, for their role in nutrient-starved organisms. Rao et al. (2008) showed that M. tuberculosis lowers its intracellular ATP level when it shifts down to the nonreplicating state due to oxygen starvation. Here we demonstrate that bacilli subjected to nutrient starvation also reduce their intracellular ATP level. This suggests that reduction of the ATP level is a common metabolic theme for the survival of non-growing tubercle bacilli. As ATP is a substrate as well as a regulator for many metabolic processes, our observation might point to a role of ATP as a global regulator to terminate growth and/or to maintain viability of the non-growing state by controlling overall metabolic activity of the bacillus. Maintaining a 
constant ATP level was demonstrated to be critical for the survival of hypoxic $M$. tuberculosis, and the respiratory enzyme Ndh-2, but not Ndh-1, was shown to be required for the maintenance of ATP homeostasis (Rao et al., 2008). Various groups have reported that tubercle bacilli are susceptible to phenothiazines, a class of known Ndh-2 inhibitors (Amaral et al., 1996; Teh et al., 2007; Weinstein et al., 2005; Yano et al., 2006). The Rubin lab demonstrated the bactericidal activity of phenothiazine compounds on nutrient-starved M. tuberculosis (Xie et al., 2005). We confirmed this result and in addition showed that Ndh- 1 is non-essential for Loebel bacilli (see Supplementary Fig. S1, available with the online version of this paper). Consistent with the requirement for Ndh-2 activity, we further demonstrated that nutrient-starved bacilli continue to consume oxygen and that an external electron acceptor is important for their survival: shifting nutrient-starved bacilli to an anaerobic environment caused death. Nitrate could substitute for oxygen as terminal electron acceptor, allowing the survival of the hypoxic, nutrient-starved pathogen. Loebel bacilli apparently can switch to nitrate respiration. Chemical probing with the ATP synthase inhibitors TMC207 and DCCD showed that oxidative phosphorylation is required for the maintenance of ATP homeostasis and viability in hypoxic non-growing $M$. tuberculosis (Rao et al., 2008). Most surprisingly, the viability of Loebel bacilli is only affected by DCCD, but not by the more specific TMC207. Both inhibitors reduced the intracellular ATP level of the nutrient-starved organism, but only DCCD led to a reduction that was large enough to kill the organism. This could indicate that Loebel bacilli have a reduced permeability for TMC207. However, further experimental data are required to elucidate the underlying molecular mechanism.

We showed for the first time in a head-to-head comparison, that nutrient-starved organisms are dramatically more drug tolerant than oxygen-starved bacilli (Table 1, top). Rifampicin for instance was 50 -fold less bactericidal to Loebel bacilli compared to Wayne bacilli, indicating a much lower transcriptional activity in the nutrient-starved bacillus. Taken together, these comparative studies show that downregulation of the ATP level, maintenance of ATP homeostasis and essentiality of Ndh-2 are common metabolic themes shared by oxygen- and nutrient-starved $M$. tuberculosis. These processes might therefore represent attractive targets for drug discovery. That ATP level reduction is indeed of functional relevance for the survival of non-replicating bacilli was indicated by the behaviour of the icl loss-of-function mutant: this mutant was not able to reduce its intracellular ATP concentration upon carbon starvation and subsequently lost viability. This establishes a first functional link between ATP level control and intermediary metabolism.

In summary, our work reveals some metabolic similarities between oxygen- and nutrient-starved tubercle bacilli: both reduce their ATP level upon entry into the non-replicating state and further maintain a low ATP level. The respiratory chain enzyme Ndh-2, but not Ndh-1, and the $\mathrm{F}_{0} \mathrm{~F}_{1}$ ATP synthase are required to maintain full viability in both models. There are also differences: nutrient-starved bacilli require either oxygen or nitrate as external terminal electron acceptor for survival, whereas hypoxic bacilli are independent of this requirement. The electron acceptor of anaerobic bacilli remains to be determined (Boshoff \& Barry, 2005; Rao et al., 2008). Finally, the death of the icl loss-of-function mutant $\Delta i c l$, which was unable to reduce its ATP level upon nutrient deprivation, suggests that reduction of the ATP level could be an essential step in the metabolic adaptation and remodelling process upon the shiftdown of M. tuberculosis to non-replicating survival.

\section{ACKNOWLEDGEMENTS}

We would like to thank Patricia Sequeira and Mai Ping Tan for stimulating discussions, Pamela Thayalan and Boon Heng Lee for technical assistance as well as Sylvie Alonso and Wenwei Lin for providing the icl complementation vector pMV306-icl. We acknowledge William Jacobs for the gift of the pYUB854 plasmid and Tanya Parish for the pGOAL17 plasmid.

\section{REFERENCES}

Amaral, L., Kristiansen, J. E., Abebe, L. S. \& Millett, W. (1996). Inhibition of the respiration of multi-drug resistant clinical isolates of Mycobacterium tuberculosis by thioridazine: potential use for initial therapy of freshly diagnosed tuberculosis. J Antimicrob Chemother 38, 1049-1053.

Andries, K., Verhasselt, P., Guillemont, J., Göhlmann, H. W., Neefs, J. M., Winkler, H., Van Gestel, J., Timmerman, P., Zhu, M. \& other authors (2005). A diarylquinoline drug active on the ATP synthase of Mycobacterium tuberculosis. Science 307, 223-227.

Bardarov, S., Bardarov, S., Jr, Pavelka, M. S., Jr, Sambandamurthy, V., Larsen, M., Tufariello, J., Chan, J., Hatfull, G. \& Jacobs, W. R., Jr (2002). Specialized transduction: an efficient method for generating marked and unmarked targeted gene disruptions in Mycobacterium tuberculosis, M. bovis BCG and M. smegmatis. Microbiology 148, 30073017.

Betts, J. C., Lukey, P. T., Robb, L. C., McAdam, R. A. \& Duncan, K. (2002). Evaluation of a nutrient starvation model of Mycobacterium tuberculosis persistence by gene and protein expression profiling. Mol Microbiol 43, 717-731.

Boshoff, H. I. \& Barry, C. E., III (2005). Tuberculosis - metabolism and respiration in the absence of growth. Nat Rev Microbiol 3, 70-80.

Cattell, K. J., Lindop, C. R., Knight, I. G. \& Beechey, R. B. (1971). The identification of the site of action of $N, N^{\prime}$-dicyclohexylcarbodi-imide as a proteolipid in mitochondrial membranes. Biochem J 125, 169177.

Dahl, J. L., Kraus, C. N., Boshoff, H. I., Doan, B., Foley, K., Avarbock, D., Kaplan, G., Mizrahi, V., Rubin, H. \& Barry, C. E., III (2003). The role of $\mathrm{Rel}_{\mathrm{Mtb}}$-mediated adaptation to stationary phase in long-term persistence of Mycobacterium tuberculosis in mice. Proc Natl Acad Sci U S A 100, 10026-10031.

Dick, T. (2001). Dormant tubercle bacilli: the key to more effective TB chemotherapy? J Antimicrob Chemother 47, 117-118.

Fenhalls, G., Stevens, L., Moses, L., Bezuidenhout, J., Betts, J. C., Helden, P. van, Lukey, P. T. \& Duncan, K. (2002). In situ detection of Mycobacterium tuberculosis transcripts in human lung granulomas 
reveals differential gene expression in necrotic lesions. Infect Immun 70, 6330-6338.

Gordhan, B. G., Smith, D. A., Kana, B. D., Bancroft, G. \& Mizrahi, V. (2006). The carbon starvation-inducible genes Rv2557 and Rv2558 of Mycobacterium tuberculosis are not required for long-term survival under carbon starvation and for virulence in SCID mice. Tuberculosis (Edinb) 86, 430-437.

Harries, A. D. \& Dye, C. (2006). Tuberculosis. Ann Trop Med Parasitol 100, 415-431.

Hinds, J., Mahenthiralingam, E., Kempsell, K. E., Duncan, K., Stokes, R. W., Parish, T. \& Stoker, N. G. (1999). Enhanced gene replacement in mycobacteria. Microbiology 145, 519-527.

Honer Zu Bentrup, K., Miczak, A., Swenson, D. L. \& Russell, D. G. (1999). Characterization of activity and expression of isocitrate lyase in Mycobacterium avium and Mycobacterium tuberculosis. J Bacteriol 181, 7161-7167.

Koul, A., Dendouga, N., Vergauwen, K., Molenberghs, B., Vranckx, L., Willebrords, R., Ristic, Z., Lill, H., Dorange, I. \& other authors (2007). Diarylquinolines target subunit $\mathrm{c}$ of mycobacterial ATP synthase. Nat Chem Biol 3, 323-324.

Koul, A., Vranckx, L., Dendouga, N., Balemans, W., Van den Wyngaert, I., Vergauwen, K., Göhlmann, H. W., Willebrords, R., Poncelet, A. \& other authors (2008). Diarylquinolines are bactericidal for dormant mycobacteria as a result of disturbed ATP homeostasis. J Biol Chem 283, 25273-25280.

Lee, M. H., Pascopella, L., Jacobs, W. R., Jr \& Hatfull, G. F. (1991). Sitespecific integration of mycobacteriophage L5: integration-proficient vectors for Mycobacterium smegmatis, Mycobacterium tuberculosis, and bacille Calmette-Guerin. Proc Natl Acad Sci U S A 88, 3111-3115.

Lim, A., Eleuterio, M., Hutter, B., Murugasu-Oei, B. \& Dick, T. (1999). Oxygen depletion-induced dormancy in Mycobacterium bovis BCG. J Bacteriol 181, 2252-2256.

Loebel, R. O., Shorr, E. \& Richardson, H. B. (1933a). The influence of adverse conditions upon the respiratory metabolism and growth of human tubercle bacilli. J Bacteriol 26, 167-200.

Loebel, R. O., Shorr, E. \& Richardson, H. B. (1933b). The influence of foodstuffs upon the respiratory metabolism and growth of human tubercle bacilli. J Bacteriol 26, 139-166.

McKinney, J. D., Honer zu Bentrup, K., Munoz-Elias, E. J., Miczak, A., Chen, B., Chan, W. T., Swenson, D., Sacchettini, J. C., Jacobs, W. R., Jr \& Russell, D. G. (2000). Persistence of Mycobacterium tuberculosis in macrophages and mice requires the glyoxylate shunt enzyme isocitrate lyase. Nature 406, 735-738.

Nyka, W. (1974). Studies on the effect of starvation on mycobacteria. Infect Immun 9, 843-850.
Ovchinnikov, Y. A.., Abdulaev, N. G. \& Modyanov, N. N. (1982). Structural basis of proton-translocating protein function. Annu Rev Biophys Bioeng 11, 445-463.

Parish, T. \& Stoker, N. G. (2000). Use of a flexible cassette method to generate a double unmarked Mycobacterium tuberculosis tlyA plcABC mutant by gene replacement. Microbiology 146, 1969-1975.

Primm, T. P., Andersen, S. J., Mizrahi, V., Avarbock, D., Rubin, H. \& Barry, C. E., III (2000). The stringent response of Mycobacterium tuberculosis is required for long-term survival. J Bacteriol 182, 48894898.

Rao, S. P., Alonso, S., Rand, L., Dick, T. \& Pethe, K. (2008). The protonmotive force is required for maintaining ATP homeostasis and viability of hypoxic, nonreplicating Mycobacterium tuberculosis. Proc Natl Acad Sci U S A 105, 11945-11950.

Stover, C. K., de la Cruz, V. F., Fuerst, T. R., Burlein, J. E., Benson, L. A., Bennett, L. T., Bansal, G. P., Young, J. F. \& Lee, M. H. (1991). New use of BCG for recombinant vaccines. Nature 351, 456-460.

Teh, J. S., Yano, T. \& Rubin, H. (2007). Type II NADH : menaquinone oxidoreductase of Mycobacterium tuberculosis. Infect Disord Drug Targets 7, 169-181.

Via, L. E., Lin, P. L., Ray, S. M., Carrillo, J., Allen, S. S., Eum, S. Y., Taylor, K., Klein, E., Manjunatha, U. \& other authors (2008). Tuberculous granulomas are hypoxic in guinea pigs, rabbits, and nonhuman primates. Infect Immun 76, 2333-2340.

Wayne, L. G. \& Hayes, L. G. (1996). An in vitro model for sequential study of shiftdown of Mycobacterium tuberculosis through two stages of nonreplicating persistence. Infect Immun 64, 2062-2069.

Wayne, L. G. \& Sohaskey, C. D. (2001). Nonreplicating persistence of Mycobacterium tuberculosis. Annu Rev Microbiol 55, 139-163.

Weinstein, E. A., Yano, T., Li, L. S. \& other authors (2005). Inhibitors of type II NADH:menaquinone oxidoreductase represent a class of antitubercular drugs. Proc Natl Acad Sci U S A 102, 4548-4553.

Williams, K. J. \& Duncan, K. (2007). Current strategies for identifying and validating targets for new treatment-shortening drugs for TB. Curr Mol Med 7, 297-307.

Xie, Z., Siddiqi, N. \& Rubin, E. J. (2005). Differential antibiotic susceptibilities of starved Mycobacterium tuberculosis isolates. Antimicrob Agents Chemother 49, 4778-4780.

Yano, T., Li, L. S., Weinstein, E., Teh, J. S. \& Rubin, H. (2006). Steadystate kinetics and inhibitory action of antitubercular phenothiazines on Mycobacterium tuberculosis type-II NADH-menaquinone oxidoreductase (NDH-2). J Biol Chem 281, 11456-11463.

Edited by: G. R. Stewart 